\title{
X-RAY LINE FORMATION IN ASTROPHYSICAL ENVIRONMENTS: THE L-SHELL SPECTRA OF IONIZED IRON
}

\author{
Duane A. Liedahl ${ }^{1}$, Steven M. Kahn ${ }^{1}$, Albert L. Osterheld ${ }^{2}$, William H. Goldstein ${ }^{2}$ \\ ${ }^{1}$ Department of Physics and Space Sciences Laboratory \\ University of California, Berkeley, CA, 94720, USA \\ ${ }^{2}$ High Temperature Physics Division \\ Lawrence Livermore National Laboratory, Livermore, CA, 94550, USA
}

\begin{abstract}
We have initiated an extensive atomic modeling effort applicable to $\mathrm{X}$ ray line emission in high-temperature astrophysical plasmas. The emphasis of our program is on the detailed accounting of the mechanisms which populate excited states of highly ionized atoms over a wide range of electron temperatures and densities. As a first demonstration we have calculated spectra for the important $L$-shell ions Fe XVIXIX in a complete collisional-radiative model under conditions appropriate to solar coronal plasmas. Using methods presented here, we have synthesized the X-ray spectra of solar flares and active regions over the wavelength interval $13-18 \AA$. The atomic model, which includes 705 atomic energy levels, is the largest and most detailed of its kind. In this introductory paper, we discuss the effects of dielectronic recombination on the spectrum of $\mathrm{Fe} \mathrm{XVI}$ and present a new technique whereby the $3 \mathrm{~s}$ lines can be used as a diagnostic of the electron temperature. Also included are new values for the rate of resonance excitation of the $n=3 \mathrm{Fe}$ XVII excited states. These rates are lower than those previously obtained and suggest that resonance excitation does not contribute significantly to the population kinetics. Finally, we present a direct comparison of our a calculated model spectrum with data from a solar flare.
\end{abstract}

\section{INTRODUCTION}

The new generation of high-spectral-resolution X-ray telescopes (AXAF, XMM) will provide data much too detailed to be properly analyzed in the context of existing plasma emission models. The current models suffer from either an insufficient treatment of the atomic physics or from their reliance on the assumption of collisional equilibrium. These deficiencies are especially apparent in the cases of X-ray binaries and active galactic nuclei where the sites of line emission are likely to be photoionized and far from collisional equilibrium so that line emissivities cannot be characterized by the local electron temperature.

We have embarked upon an atomic modeling program to develop plasma diagnostics for application to the phenomenology of cosmic X-ray sources. Beyond the augmentation of plasma emission models commonly in use, our program will extend diagnostic techniques to a much wider variety of astronomical environments. 
Motivated by the high quality of extant solar X-ray data and the need to establish a point of reference for our model spectra, the subject of our first study is the $L$-shell spectrum of iron under solar flare conditions. Line emission from the ions of interest, Fe XV-XXIV, are known to comprise major portions of the X-ray spectra of solar flares and solar active regions (e.g., Phillips et al. 1982). Indeed, nearly the entire solar flare spectrum from 14 - $18 \AA$ can be synthesized from Fe XVI - XIX (see Figure 1). Since the Fe XVII $L$-shell lines are especially prominent features in the soft $\mathrm{X}$-ray band of many cosmic sources, a considerable amount of effort has gone into a careful analysis of the relevant atomic physics. Comparably detailed analyses of the remaining Fe $L$-shell ions are underway. We are extending our calculations to the low-density, multi-keV temperature regime appropriate to young supernova remnants. Also, processes involving an external source of ionizing radiation and the creation of highly excited $1 s$-hole states are now being coupled into the code in order to address the problem of line formation in accretion-powered X-ray sources.

\section{THE MODEL}

Only a brief description of the model will be given here. The atomic structure, radiative transition rates, and collisional rates are computed using a sophisticated atomic physics package originated by $\mathrm{M}$. Klapisch and A. Bar-Shalom of Hebrew University (Klapisch 1971; Klapisch et al. 1977; Bar-Shalom et al. 1988). The atomic structure is computed $a b$ initio using the relativistic, multi-configuration, parametric potential method. The collisional rates are computed in the relativistic distorted wave approximation. The model is collisional-radiative, as opposed to coronal, i.e., all possible level-to-level transition rates, collisional and radiative (e1, e2, $\mathrm{m} 1$, $\mathrm{m} 2$ ), are calculated and included in the rate equations. The model consists of all singly excited levels of Fe XVIII and Fe XIX for $\mathrm{n}=3$, all singly excited levels of $\mathrm{Fe}$ XVII for $\mathrm{n}=3$ and 4 , all doubly excited levels of $\mathrm{Fe}$ XVI of the form $2 p^{5} 3 l 3 l^{\prime}$ and $2 s 2 p^{6} 3 l 3 l^{\prime}$, and the set of configurations of the form $2 p^{6} 3 l 3 l^{\prime}$ of Fe XV for a total of 705 levels.

Excited states can also be populated by processes involving adjacent ion stages. Included are the results of detailed calculations of dielectronic recombination into excited levels of Fe XVII originating from the three levels of the Fe XVIII ground configuration and summed over the Fe XVII $2 p^{4} 3 l 3 l^{\prime}, 2 p^{4} 3 l 4 l^{\prime}$ and $2 s 2 p^{5} 3 l 3 l^{\prime}$ doubly excited states (Chen 1988). The Fe XVI model includes the 237 doubly excited levels of the form $2 p^{5} 3 l 3 l^{\prime}$. We have included the satellites to Fe XVII resonance lines which are formed by radiative relaxation of these doubly excited states (Oreg 1988). Resonance excitation of the $n=3$ singly excited states of $F e$ XVII begin with a radiationless electron capture onto the Fe XVII ground state into a doubly excited state of Fe XVI ( $3 \ln l^{\prime}$ for $n=6-14$ and $2 p^{5} 4 l 4 l^{\prime}$ ) which then autoionizes to an Fe XVII singly excited state. New rates for this process have been calculated (Goldstein 1988) and included in the rate equations. 


\section{RESULTS}

Earlier theoretical treatments of the Fe XVII spectrum (e.g., Smith et al. 1985) have not accounted for the effects of dielectronic recombination from Fe XVIII. We have found that, in addition to regulating the ionization balance at coronal temperatures, dielectronic recombination can have a pronounced effect on the line emission. For example, at $T_{e}=250 \mathrm{eV}\left(\log T_{e}=6.45\right)$, approximately one-third of the flux into Fe XVII excited states originates from the ground state of Fe XVIII by way of dielectronic recombination. Thus, Fe XVII levels which are populated substantially by cascade, such as $2 s^{2} 2 p^{5} 3 s$, can be strongly coupled to the ground configuration of $\mathrm{Fe}$ XVIII. However, the collisionally populated $2 s^{2} 2 p^{5} 3 d$ levels are largely unaffected by processes involving the Fe XVIII population. Consequently, the nine Fe XVII line ratios of the form $I(3 s) / I(3 d)$ must be treated as functions of both $T_{e}$ and $n_{1}^{F} / n_{1}$. Let us refer to the latter quantity as $R$ and define a function, $\Phi$, such that $I(3 s) / I(3 d)=\Phi\left(T_{e}, R\right)$ for a given pair of lines.

By taking the ratio of two collisionally excited lines from neighboring ion stages, one can obtain a simple temperature-dependent expression for the relative ion fractions. The most useful lines of this kind are the Fe XVII $3 d$ line at $15.01 \AA$ and the Fe XVIII two-line blend at $-14.20 \AA$. One obtains an expression of the form $R\left(T_{e}\right)=$ $\Gamma\left(T_{e}\right) \times I(14.20) / I(15.01)$. The function, $\Gamma\left(T_{e}\right)$, involves collisional rate coefficients and a rate coefficient which describes the rate of production of unresolved dielectronic recombination satellites to the $15 \AA$ resonance line. The importance of this latter effect has been pointed out by Raymond and Smith (1986). We are presently performing more detailed calculations of this process. Simultaneous solution of the expressions for $\Phi\left(T_{e}, R\right)$ and $R\left(T_{e}\right)$ will yield both the electron temperature and the relative ionization fractions of Fe XVII and Fe XVIII. Of course, this assumes that the emitting regions of the two ions are spatially coincident. In principle, a similar method can be used for the remaining ion stages.

New calculations of resonance excitation (Goldstein 1988) of the $2 p^{5} 3 s$ states of Fe XVII suggest that this process is not as effective at populating these levels as had been predicted by previous calculations (e.g, Smith et al. 1985). Using the old rates, temperatures inferred from line ratios of the form $I(3 s) / I(3 d)$ are too high since the rate for resonance excitation is a decreasing function of temperature. However, if dielectronic recombination from Fe XVIII is also accounted for, the effect on the temperature estimate can be partially offset. This effect manifests the temperature dependence of the collisional excitation rate coefficients, rather than the dielectronic rate coefficients which do not change appreciably over the temperature range of interest. In other words, for a given $R$ (see above), one generally expects ratios of the form $I(3 s) / I(3 d)$ to decrease with temperature because the excitation rates for the collisionally populated $3 d$ levels are increasing over the solar coronal temperature range.

Finally, we present a model spectrum for comparison with a spectrum from a solar active region (Figure 1, Rugge and McKenzie 1985). We defer quantitative conclusions to a later paper (Liedahl, et al. 1988) where the unresolved satellites to the 
$15 \AA$ will be accounted for. For the sake of discussion, the model spectrum was calculated with $T_{e}=250 \mathrm{eV}$ and $n_{e}=10^{11}$. Note that this part of the spectrum contains an important contribution from O VIII Lyman $\beta$ at $16 \AA$ which has not been included in our model.
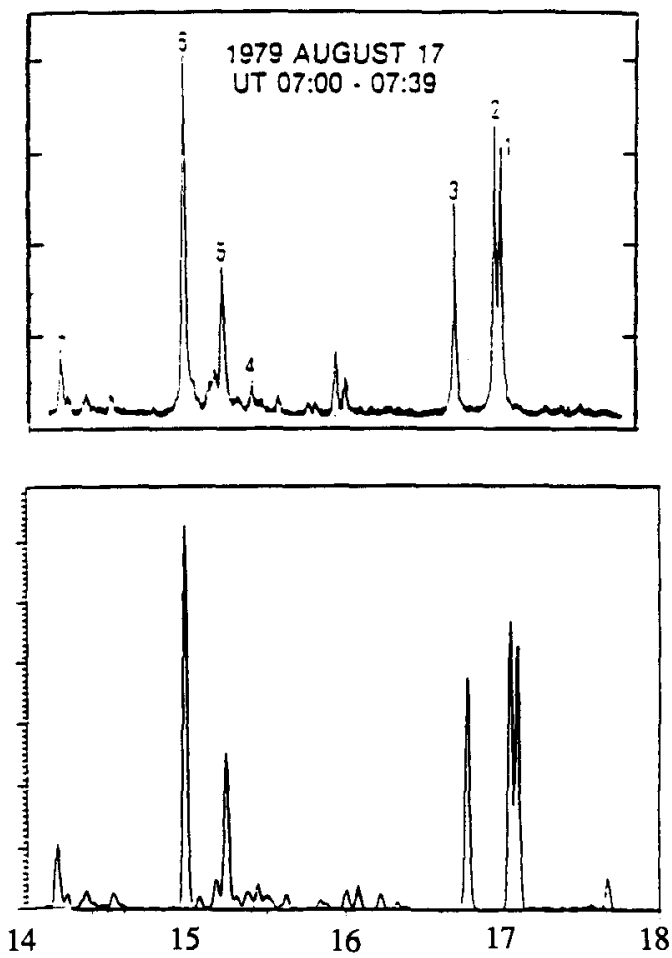

Figure 1. Top: Solar flare spectrum (Rugge and McKenzie 1985). The lines numbered 1-6 are Fe XVII lines and line 7 is the Fe XVIII two-line blend referred to in the text. Bottom: Model spectrum with $T_{e}=250 \mathrm{eV}$ and $n_{e}=10^{11} \mathrm{~cm}^{-3}$.

\section{REFERENCES}

Bar-Shalom, A., Klapisch, M., Oreg, J. 1988, Phys. Rev. A 381773. Chen, M. 1988, private communication.

Goldstein, W.H., This volume and Talk presented at the IAU Collog. No. 115 on "Recent Advances in Atomic Modeling", Cambridge, Massachusetts, USA, Aug 1988. Klapisch, M. 1971, Computer Phys. Comm. 2, 239.

Klapisch, M., Schwab, J.L., Fraenkel, B.S., and Oreg, J. 1977, J. Opt. Soc. Am. 61 148.

Liedahl, D.A., Kahn, S.M., Goldstein, W.H., and Osterheld, A.L. 1988, in preparation. Oreg, J. 1988, private communication.

Phillips, K.J.H., et al. 1982, Ap. J., 256, 774.

Raymond, J.C., and Smith, B.W. 1986, Ap. J., 306, 762.

Rugge, H.R., and McKenzie, D.L. 1985, Ap. J., 297, 338.

Smith, B.W., Raymond, J.C., Mann, J.B., and Cowan, R.D., 1985, Ap. J., 298, 898. 